\title{
Automated Architecture: Why CAD, Parametrics and Fabrication are Really Old News
}

\author{
Alfredo Andia, Ph.D. \\ Florida International University, USA \\ andia@post.harvard.edu
}

\begin{abstract}
Automation is transforming a significant number of industries today. This paper discusses how the Design and Construction industry is also entering into a new era of automation. In the paper I observe that designers are automating by using parametric tools (BIM, scripting, etc.) while contractors are moving into pre-fabrication and modularization. Both conceptualizations are incomplete. The paper presents how we are in the first steps of creating learning algorithms that develop specific intelligence in design synthesis and how the design field will became even more sophisticated as a second generation of multi-material 3D printing techniques produce new materials.
\end{abstract}

Keywords: Automation; Architectural design; Artificial intelligence; Learning algorithms; Multi-material printers.

\section{Introduction}

This paper is organized around the divergent automation narratives that Designers and Contractors are having today. There are four major ideas. In the first part the paper shows that the most advanced automation narratives by Architects/Engineers is related to the use of parametric software. In the second part the paper I argue that parametric will not be able to automate design to a significant level. Instead machine learning algorithms offer a more advanced paradigm for design.

Third, I show how contractors are moving quickly into prefabrication and modularization. In the fourth section of the paper I argue that pre-fabrication and modularization ideas will be challenged by multi-material 3D printers that will allow us to design new materials at the macroscopic and microscopic level. Since traditional design skills will not be able to design materials at the macro and micro level machine learning algorithms will become more critical in the design process.

\section{Automating Design via Paremetricism}

The possibilities of automating design synthesis process have moved into the forefront discourse of practice as architects and engineers have begun to use parametric software in the past decade. The most basic conceptualization of parametric refers to a 3D digital model associated to knowledge structures, information, performance properties, and automatic procedures that can aid the designer to construct quick scenarios during design. These models can be updated overtime and reused. Ideally, if more parameters are increasingly included they can further breed its associations to all kinds of data, performance parameters, procedures, and knowledge. I will argue in the next section that this paradigm is overly optimistic and after more than 40 years of endeavors we can say that it will fail to achieve its main automation goals.

\section{Three parametric paradigms today}

As 3D parametric software and tools are being rediscovered by architectural firms they are beginning to change their design workflows. Contemporary design practices have developed at least three different narratives with regards to parametric design.

Parametric Formalism: parametric modeling and scripting has been used to find intricate utopian/dystopian formal visions in studios usually led by professors that are closely linked to the paperless studio digital avant-garde that emerged in the 90s and 00s. Architects using this narrative use parametric techniques to substitute the sculptural or figurative designer in developing complex spatial formation (Schumacher, 2009).

Parametric BIM: BIM has become one of the central themes in the computerization of Architectural practice today. BIM software and processes allow architects to construct virtual models that accurately replicate building systems and materials. The merging of these parametric BIM models with embedded sensors procurement procedures, intelligent 3D libraries, price engines, and bidding systems will move the narrative further. However, despite the exaggerated claims that BIM is revolutionizing construction, BIM is still very manually intensive and it is not be a radically more automated method.

Meta-Heuristic Parametric: A third type of narrative about parametric design is beginning to emerge in research and development units inside large design firms. Many of these units are developing generative procedures that can integrate certain design workflows with interactive modeling tools and environmental benchmarking or structural procedures (Coates, 2010). Groups such as the Research \& Development Group at Aedas, Blackbox at SOM, and the Advanced Geometry Unit at Arup are some examples of designers exploring generative and analytical computational processes in design (Derix, 2010). 


\section{Automating Design via Machine Learning}

As described above scripting and parametric projects has been developed with relative success in a number of places in architectural academia and practice in the past decade. However, these endeavors are far from fully automating major design workflows in the AEC industry. Those that optimistically advocate parametricism imply that the more parameters are programmed in a digital model the more automated the design process will became. However, the more factors you include in a parametric model the exponentially more difficult it becomes to connect design associations.

\section{Parametric: first stage of Al}

In the first stage, the most prehistoric stage of $\mathrm{Al}$, one can find Parametric systems that via parameter adjustments or combinatorial search do basic intelligent operations. Winograd and Flores mention that after a short-lived peak in the 1950s and 1960s parametric work had been almost fully abandoned in the computer science community. Parametric allows for the coding of human reasoning however it always requires the hand of a coder that is able to observe all the potential steps of every condition of intelligent behavior and eventually can not fully automate large workflows of human flow.

\section{Computers as autopoietic self-organizing systems}

Terry Winograd and Fernando Flores wrote in 1987 a book called "Understanding Computers and Cognition: A New Foundation for Design" (Winograd \& Flores, 1987). The book has proven to be deeply influential in Computer Design Theory however it is hardly known in architectural or engineering design circles. They place forward the notion that the design of Artificial Intelligence systems will come in three different stages of computer learning: 1. Parametric; 2. Machine Learning; and 3. General Artificial Intelligence. We will refer to only 1 and 2 as General Artificial Intelligence is far from being an achievable in at least the next 2 decades.

\section{Machine learning: second stage of Al}

A second level of impacts of artificial intelligence in Architecture occurs when computers perform concept learning and concept formation. Here algorithms learn from data. These learning algorithms find patterns in data and build probability or predictive models for a specific job. Today learning algorithms work in a large array of tasks such as translating text, spam detection, personalized web ads, drug design, medical diagnosing, stock trading, and in many other tasks. For example, translation algorithms do not understand text but if they are feed two texts in two different languages it will be able to detect patterns such as that the word "one" in english is "uno" in spanish. The more text is feed to the algorithm the higher certainty its predictive model will have to find the right translation.
Arthur Samuel, one of the early pioneers of $\mathrm{Al}$, described machine learning as the "field of study that gives computers the ability to learn without being explicitly programmed" (Ratner, 2000). Automated learning systems learn from data using techniques such as artificial neural networks, decision tree learning, support vector machines, bayesian networks, boltzmann machines among many others. Machine learning algorithms can be classified into several types depending on the desired results and data available. They can be categorized as: supervised learning, unsupervised learning, semi-supervised learning, reinforcement learning, learning to learn, or transduction. An example of supervised learning is a driverless car that is trained how to drive by creating a neural network system that captures images of the road and at the same time records the steering directions of human drivers. Once the system is trained the car will capture images of the road as it moves and the steering direction will be controlled by the neural network optimized results ( $\mathrm{Ng}, 2009)$.

Today, learning algorithms are everywhere. They are automatically selecting companies for venture capital firms and they are automating the discovery processes of many large practices in the legal community. Complex algorithms are already replacing engineers in tasks such as chip design, substituting journalists in writing sport news, grading English essays, developing patrol routes for the Los Angeles police, and IBM's Watson supercomputer beat human competitors at "Jeopardy!" just after two years of training.

\section{Learning algorithms in architectural design}

Machine learning algorithms in the architectural design domain can evolve in several directions. These systems can be trained by feeding them a list of program, size and adjacencies. Which are very different to the rule based programmed or parametric system found in previous work from Per Galle (Galle, 1981) or Bill Mitchell (Mitchell, 1990). More advanced system will receive data from floor, plans, elevations and models. Initially floor plans can be obtained from probability optimization from which threedimensional models can be build based on different architectural styles. These learning algorithms have allowed for the automated generation of residential houses floor plans, sections and 3D model based on data feed from a book. A more sophisticated type of method can be developed by training the machine learning algorithms to become skilled at styles or design concepts found in a diverse population of work and designers. Style as creative freedom is a higher level semantic problem found in design and initial ground have been developed in drawing and painting algorithms (Lindemeier, 2013).

\section{Automating Construction via Prefab and Modularization}

Most of the parametric discourse emerging in Architecture today and described above belongs to the design automation type. However, from Construction/Engineering side many other ideas 
have also emerged and materialized. The Japanese construction industry since the late 1980s leaded the world in construction automation. By 1999 the Japanese construction industry could account for more than 550 systems for unmanned operation and automation of construction and civil engineering operations (Obayashi, 1999). Today research centers in Japan have advanced investigations that test and use humanoids robots in construction. Despite these advances fully automated Japanese construction systems have failed to produce significant gains and are hardly exportable to other countries.

\section{Pre-Fabrication and modularization in the US}

Most of the computerization metaphors found in Japanese construction projects are related to move automation into the construction site. Instead, pre-fabrication and modular construction in the US, Europe, and particularly China seem to offer a more viable vision for the automation of construction. In the past 3-to-5 years, a significant number of construction sites in the US have become increasingly assembly sites in which elements such as HVAC systems, wall units, even restrooms components are pre-fabricated off site reducing safety, cost, waste, and the schedule of projects. In $200973 \%$ of Contractors surveyed in the US believed that BIM would allow them to increase prefabrication (McGraw Hill, 2009). Another study from 2011 that surveyed contractors that are using pre-fabrication and modularity report that $66 \%$ of project schedules have been reduced, $35 \%$ by 4 weeks or more, and $65 \%$ project budget have been decreased, $41 \%$ by $6 \%$ or more.

\section{Pre-Fabrication and Modularization in China}

In China the Company Broad Group has stunned the category of pre-fabrication and modular construction by completing several buildings in record time. In 2010 they built a 6 story pavilion for the Shanghai Expo in 1 day and in 2011 they assembled and completed a 30 floors hotel in 15 days. The Broad Group has developed since 2009 a modular pre-fabrication system in which around $93 \%$ of the labor-hours of construction are spent in a factory - compared to just around $40 \%$ which is traditionally obtained in the west. The time spend at the construction site is minimal thus reducing construction errors, delay, accidents, construction site pollution, and site waste.

The Broad Group claims that by controlling the construction via pre-fabrication and introducing their energy equipment they can also improve significantly the energy efficiency of their buildings, better control its life cycle - greatly reducing $\mathrm{CO} 2$ emissions. Broad Group claims that their latest 17,000 sq. $\mathrm{mt}$. hotel constructed in 2011 is 5 times more energy efficient than traditional buildings, can resist a magnitude 9 earthquake, and that the air inside the building is 20 times purer than outdoor air.

\section{Automating Construction via the Future of 3D Printing}

The extraordinary case of Broad Group is potentially open to more advanced factory automation narratives such as robotics, warehouse mechanization, and manufacturing computerization. In this section I argue that the factory automation narratives, although extraordinary in the construction industry, are still narrow. Here we look at how 3D printing is eventually challenging the way we will design and manufacture materials for construction.

The first thing to understand about $3 D$ printers is that it is a technology that is not going away. What we understand today for $3 \mathrm{D}$ printing it is just the first generation of the technology. This is a technology that is growing explosively in many different directions. It emerged in the 1980s initially as a computerized additive manufacturing processes to produce 3D objects. However a second generation of multi-material printers is allowing us to create a completely new array of materials.

\section{D printing new multi-materials}

A more disruptive era of $3 d$ printing will arrive as these systems advance to produce new materials. A newer generation of multimaterial printers are printing patterns of materials at the microscale level. The properties of these new mixed materials are completely new.

Hod Lipson and Melba Kurman give an example. They say that if you stretch a rubber band you see that the rubber band gets thinner as you stretch it more. That is called Poisson effect or Poisson ratio. They printed a material that had a negative Poisson ratio. That means that the material of a rubber band becomes thicker as it gets stretched (Lipson \& Kurman, 2013). This peculiar material that can not be found in nature is called Auxetic. Auxetic materials can not easily be manufactured with traditional methods but can be printed on demand by high-resolution multi-material printers. Lipson and Kurman state that Auxetic materials can be used in car bumpers that when hit it can direct the force of the crash to other parts of the vehicle - protecting the passengers. Multi-materials printed in different patterns might also achieve more optimal behaviors than traditional materials in the construction industry.

The advent of new multi-materials printers also implies that the designer and the design process will have to change too. Traditional design processes that use CAD or BIM are usually unable to plan for material performance at the macroscopic and microscopic level. Design automation will have to play an increasingly important role in design synthesis for the construction elements that use multi-materials. Hiller and Lipson from Cornell University have presented a genetic algorithm method that is used to optimize the geometry of a multi-material cantilever beam. These authors state that the genetic algorithms are "suitable for designing the complex multi-material objects that have recently 
become possible to fabricate using additive manufacturing techniques... Instead of designing an object using traditional CAD programs, genetic algorithms allow an engineer to simply set highlevel goals to be fulfilled and the blueprint is autonomously generated" (Hiller \& Lipson, 2009).

\section{Conclusion}

In this paper I attempted to move forward a workable narrative about how the AEC industry is beginning to automate its workflows. I argue that there are two different narratives emerging in the forefronts of automation today. On the one hand we look at how a large number of Architectural and Engineering firms are transforming their practices by using Parametric, BIM, and scripting tools that help them automate parts of their design and analytical routine work from Design to Fabrication. On the other hand we observe how large Engineer/Contractors have begun to transform their construction practices by moving gradually into pre-fabrication, modularization, and manufacturing.

I argue that both narratives are incomplete. The design automation lead by Architects and Engineers using parametric algorithms will not succeed in automating a significant number of workflows. Instead I argue that machine learning algorithms such as the one used in many other industries will allow the design fields to automate their processes in a more effective way than parameter adjustments.

We also argue that the current trend from Engineers/Contractors for pre-fabrication and modularization will potentially encounter the raise of 3D multi-materials printers. This printers can produce all type of new materials which can be designed at the micro-level to respond to very particular performative conditions.

The advent of a more precise way of constructing will eventually lead to a transformation of the designer and the traditional design process. Traditional design processes are unable to plan with material performance at the macroscopic and microscopic level. Machine learning design automation will have to play an increasingly important role in design synthesis for the construction elements that use multi-materials in the near future.

\section{References}

Coates, P. (2010). Programming.Architecture. London: Routledge.

Derix, C. (2010). Mediating Spatial Phenomena through Computational Heuristics. in ACADIA 10: LIFE In:formation.

Galle, P. (1981). An algorithm for exhaustive generation of building floor plans. Communications of the ACM 24(12), 813-825.

Hiller, J. D. \& Lipson, H. (2009). Design automation for multi-materia printing." Solid Freeform Fabrication Symposium (SFF'09).

Lipson, H. \& Kurman, M. (2013). Fabricated: The New World of 3D Printing. Indiana: John Wiley \& Sons.

Mcgraw Hill (2009). Smartmarket Report. The Business Value Of BIM Getting Building Information Modeling To The Bottom Line. Mcgraw Hill.

Mitchell, W. J. (1990). The logic of architecture: Design, computation, and cognition. MIT press.

Lindemeier, T., Pirk, S. \& Deussen, O. (2013). Image stylization with a painting machine using semantic hints. Computers \& Graphics, 37(5), 293-301.

$\mathrm{Ng}$, A. S.U. (2009). Video Lecture - An application of supervised learning Autonomous driving, CS229 - Machine Learning. Engineering, University of Stanford.

Obayashi, S. (1999). Construction Robot System Catalogue in Japan, Japan Robot Association, Tokyo: Council for Construction Robot Research.

Ratner, B. (2000). A comparison of two popular machine learning methods. Mine Tech.

Schumacher, P. (2009). Parametricism - A New Global Style for Architecture and Urban Design. AD Architectural Design, 79(4).

Winograd, T. A., \& Flores, C. F. (1986). Understanding computers and cognition: A new foundation for design. Intellect Books. 